demonstrate the power of a well-designed study based on large samples, the data they used were recorded by many researchers and at varying levels of detail, raising the possibility of methodological biases.

Lastly, the causes of the injuries could provide some elusive insights into behaviour, activities or social norms in the past. From the shape, location and extent of traumatic injuries in skeletons, and characteristics such as the sharpness of fracture edges or the degree to which injuries had healed, it is sometimes possible to establish the most likely cause of a trauma - for example, whether the injury probably arose as a consequence of a hunting accident $^{3}$, interpersonal violence ${ }^{10}$ or intergroup conflict ${ }^{11}$. Moreover, surviving severe trauma might indicate that the injured person was cared for by members of their society ${ }^{12}$. Establishing the likelihood of each of these scenarios among Neanderthals and early modern humans will no doubt continue to challenge scientists for many years to come.

Marta Mirazón Lahr is in the Department of Archaeology, Leverhulme Centre for Human Evolutionary Studies, University of Cambridge, Cambridge CB2 1QH, UK. e-mail:mbml1@cam.ac.uk

1. Beier, J., Anthes, N., Wahl, J. \& Harvati, K. Nature 563, 686-690 (2018).

2. Berger, T. D. \& Trinkaus, E. J. Archaeol. Sci. 22 841-852 (1995).

3. Churchill, S. E. Evol. Anthropol. 11, 185-186 (2002).
4. Redfern, R. C. Injury and Trauma in Bioarchaeology. Interpreting Violence in Past Lives (Cambridge Univ. Press, 2017)

5. Lovell, N. C. in Biological Anthropology of the Human Skeleton (eds Katzenberg, M. A. \& Saunders, S. R.) 341-386 (Wiley, 2008)

6. Underdown, S. J. Period. Biol. 108, 485-493 (2006).

7. Pinker, S. The Better Angels of Our Nature: A History of Violence and Humanity (Penguin, 2011).

8. Trinkaus, E. J. Archaeol. Sci. 39, 3691-3693 (2012).

9. Lévêque, F. \& Vandermeersch, B. C. R. Acad. Sci. 291, 187-189 (1980)

10.Zollikofer, C. P. E., Ponce de Léon, M. S., Vandermeersch, B. \& Lévêque, F. Proc. Natl Acad. Sci. USA 99, 6444-6448 (2002).

11. Mirazón Lahr, M. et al. Nature 529, 394-398 2016).

12.Spikins, P., Needham, A., Tilley L. \& Hitchens, G. E. World Arch. https://doi.org/10.1080/00438243.20 18.1433060 (2018).

This article was published online on 14 November 2018.

\section{A glimpse into the heart of a quasar}

of electromagnetic radiation coming from a single point to infer the structure and dynamics of the gas and dust around the black hole. Such properties include colour, time variability, polarization and phase - the offset of an electromagnetic wave from a given position.

For the past 30 years, our best understanding of gas in the vicinity of a quasar's black hole has come from a method called reverberation mapping, which uses echoes of light (analogous to those of sound) to map out regions near the black hole ${ }^{5}$. The accretion disk emits light in all directions, some of which is observed directly by telescopes, and some of which illuminates a region of surrounding gas, known to astronomers as the broad-line region. Optical-reverberation mapping measures how long it takes the broad-line region
W hen the astronomical object 3C 273 was detected $^{1}$, to most optical telescopes it looked just like a star in our Galaxy. But in 1963, astronomers discovered $^{2,3}$ that the object was shining from a distance of 750 megaparsecs (2.4 billion light years). Whatever this mystery object was, it was producing more radiation than a trillion stars, from a region no bigger than the Solar System. Objects such as 3C 273 are now known as quasars and are understood to be powered by hot gas and dust feeding into a supermassive black hole through a structure called an accretion disk. Fifty-five years after that remarkable discovery, 3C 273 is back in the limelight. On page 657, the GRAVITY Collaboration ${ }^{4}$ reports observations of the spatially resolved rotation of hot gas in the quasar at distances much closer to the black hole than were previously possible.

A quasar can produce more energy than the entire galaxy in which it resides. Although the basic mechanism that powers a quasar is known, the anatomy of the supermassive black hole and its surroundings is not well understood. Where does the gas that feeds the black hole come from? And what effect does the resulting intense radiation have on the environment around the black hole? The findings of the GRAVITY Collaboration provide a way to answer these fundamental questions.

Determining the structure of a quasar is difficult because the black hole is extremely small and far away from Earth, and therefore the gas orbiting close to the black hole cannot be directly imaged using telescopes. Instead, astronomers rely on the properties

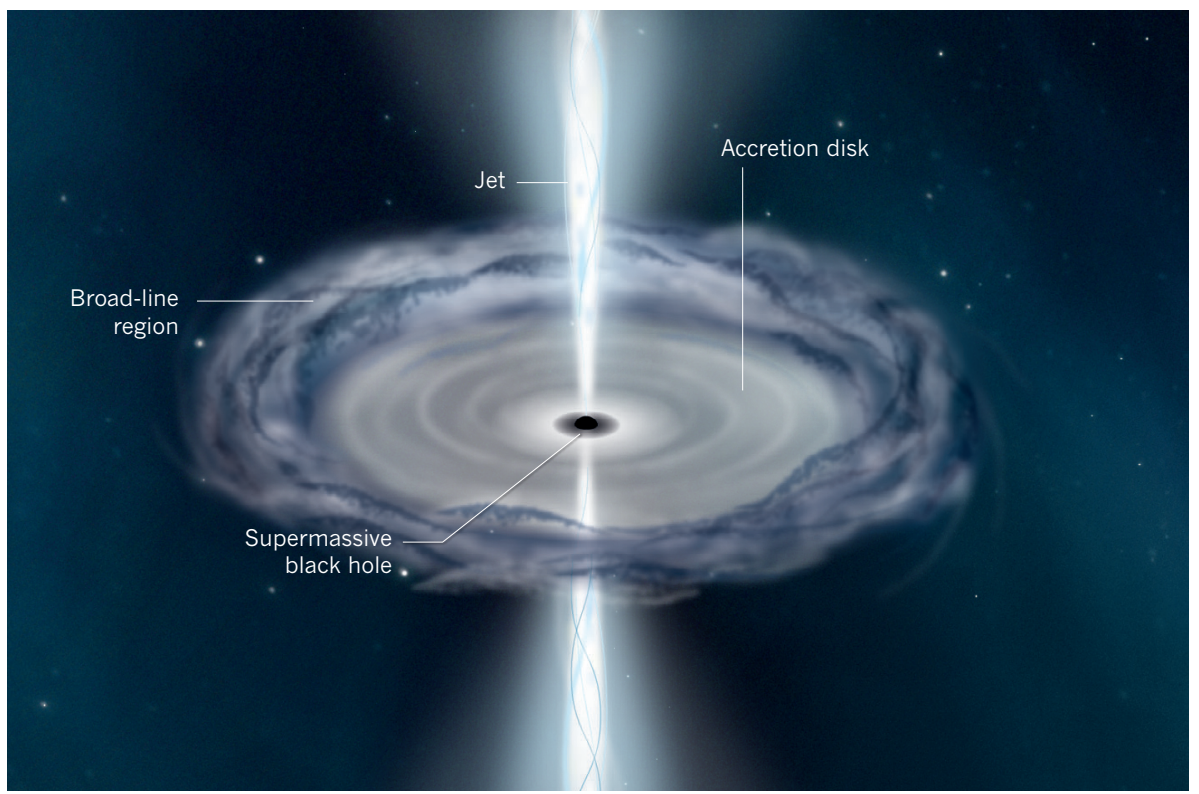

Figure 1 | Structure of the quasar 3C 273. Quasars are astronomical objects comprising a supermassive black hole surrounded by hot gas and dust. As this material is pulled towards the black hole through a structure known as an accretion disk, energy is released in the form of light and, in the case of the quasar 3C 273, as a beam of charged particles called a jet. The GRAVITY Collaboration ${ }^{4}$ reports a technique that enables the rotation of gas in a part of $3 \mathrm{C} 273 \mathrm{known}$ as the broad-line region to be spatially resolved. The researchers determine that this gas moves perpendicular to the jet and has the shape of a thick ring. 
to respond to illumination from the accretion disk, which, in effect, measures the distance between the disk and the surrounding gas ${ }^{6}$. In a similar way to how bats use echolocation to map out a dark cave, astronomers measure light echoes to map out the hot gas around black holes.

The GRAVITY Collaboration has ushered in an alternative technique that spatially resolves the motion of such gas using the GRAVITY instrument in Chile ${ }^{7}$. This instrument is an interferometer that combines the light from four near-infrared telescopes that are 8 metres in diameter to produce a virtual 'super telescope' that is $130 \mathrm{~m}$ in diameter. Because the spatial resolution of a telescope depends on its size, the use of the GRAVITY instrument is a giant step in imaging capability. The collaboration measured the offset in phase between the direct emission of light from 3C 273 and the light from the broad-line region to spatially resolve the motion of this gas in a distant quasar for the first time.

The team observed a velocity gradient in the gas on size scales of 10 microarcseconds - an achievement that is comparable to seeing a coin on the Moon from Earth. The researchers found that the motion of this gas is perpendicular to the known large-scale jet (a beam of charged particles) projected from 3C 273 (Fig. 1). The results suggest that the gas is in the form of a thick ring with a radius of 0.12 parsecs, rotating around a black hole that has a mass 300 million times that of the Sun. These findings support previous estimates from reverberation mapping of 3C 273 that indicated a similar black-hole mass and gravitationally bound gas at a distance of 0.08-0.34 parsecs from the black hole ${ }^{8,9}$.

For astronomers, the excitement about the current work is not because the results have fundamentally changed our understanding of quasars, but rather because this impressive technological advance enables an independent cross-check of optical-reverberation mapping - the most widely used method for determining the structure of gas around supermassive black holes. Optical reverberation has been measured in roughly 60 quasars $^{10}$, and the inferred properties of the gas strongly correlate with the luminosity of the quasar and the mass of the central black hole.

These correlations have been applied to large samples that comprise thousands of quasars. They have thereby informed our understanding of far-reaching aspects of astronomy, from the co-evolution of black holes and galaxies over cosmic time to the rate at which the expansion of the Universe is accelerating. Having an independent cross-check from spatially resolved interferometric observations, as reported by the GRAVITY Collaboration, is valuable for confirming several key findings in astrophysics that rely on the robustness of reverberation-mapping results.

It is important to keep in mind that the results presented in the paper are based on one particular quasar. The GRAVITY Collaboration observed 3C 273 because it was the best target for optical interferometry. However, the quasar is by no means the best target for reverberation mapping, which makes it difficult to compare the results from these two methods critically.

Going forward, the GRAVITY instrument should be capable of spatially resolving the dynamics and orientations of the broadline region in about ten other quasars ${ }^{11}$. To best corroborate or dispute sizes and structures inferred from reverberation mapping, coordinated campaigns on the same quasars using two independent techniques must be carried out. The GRAVITY instrument is at the beginning of its scientific operations, and these early technical achievements bode well for future investigations that peer deeper into the hearts of quasars.
Erin Kara is in the Department of Astronomy, University of Maryland, Maryland 20742, USA. e-mail:ekara@astro.umd.edu

1. Edge, D. O., Shakeshaft, J. R., McAdam, W. B., Baldwin, J. E. \& Archer, S. Mem. R. Astron. Soc. 68, 37-60 (1959).

2. Hazard, C., Mackey, M. B. \& Shimmins, A. J. Nature 197, 1037-1039 (1963).

3. Schmidt, M. Nature 197, 1040 (1963)

4. GRAVITY Collaboration. Nature $563,657-660$ (2018).

5. Blandford, R. D. \& McKee, C. F. Astrophys. J. 255, 419-439 (1982).

6. Peterson, B. M. Publ. Astron. Soc. Pacif. 105 247-268 (1993)

7. GRAVITY Collaboration. Astron. Astrophys. 602, A94 (2017).

8. Kaspi, S. et al. Astrophys. J. 533, 631-649 (2000)

9. Zu, Y., Kochanek, C. S. \& Peterson, B. M. Astrophys. J. 735, 80 (2011).

10.Bentz, M. C. \& Katz, S. Publ. Astron. Soc. Pacif. 127, 67-73 (2015)

11. Rakshit, S., Petrov, R. G., Meilland, A. \& Hönig, F. Mon. Not. R. Astron. Soc. 447, 2420-2436 (2015).

\section{MICROBIOLOGY}

\section{A bacterium's enemy isn't your friend}

The bacterium Staphylococcus aureus is a leading cause of hard-to-treat human infections. It now seems that, if the bacterium is infected by a virus, a viral enzyme helps the microbe to evade detection by the immune system. SEE LETTER P.705

\section{MICHAEL S. GILMORE \& ONA K. MILLER}

$\mathrm{M}$ icroorganisms thrive on our body's surfaces. The species present are not just a random assembly; rather, they are a community of organisms that are particularly well adapted to the local conditions of temperature, moisture, nutrient availability and host defences ${ }^{1}$. Staphylococcus aureus is one of our most common bacterial residents. It usually lives in nasal, respiratory and reproductive tissues without causing disease, yet, unlike many other resident bacteria, S. aureus has the capacity to give rise to a potentially deadly infection ${ }^{2}$.

During the past 50 years ${ }^{2}$, the resistance of $S$. aureus to antibiotics has become an increasing problem, and strains of the bacterium termed methicillin-resistant $S$. aureus (MRSA), which are resistant to treatment with the antibiotic methicillin and other methylated penicillin-based antibiotics, cause both hospital- and community-acquired infections around the globe. On page 705, Gerlach et al. ${ }^{3}$ describe a previously unknown mechanism whereby viruses influence whether MRSA is recognized by the immune system, shedding light on a process that might tip the balance in determining whether this bacterium will be harmless or disease-causing.

Staphylococcus aureus belongs to the
Gram-positive group of bacteria, and has been described as existing on the borderline between being a normal human microbial resident and a disease-causing organism ${ }^{4}$. This bacterium seems to have the capacity to probe for signs of host weakness, such as reduced immune defences caused by disease. When this is detected, the bacterium can increase its population to a level that can cause the death of the host ${ }^{5}$. Factors that regulate host-microbial interactions are complex, and in addition to host defences, such interactions can be influenced by the presence or absence of other bacteria ${ }^{6}$. Gerlach and colleagues report that viruses can also be part of the mix that influences host-microbial interactions in the context of MRSA.

In Gram-positive bacteria, the cell wall contains polymers known as wall teichoic acids (WTA), which are made up of ribitol phosphate or glycerol phosphate molecules and can constitute up to half of the cellwall mass ${ }^{6}$. Unlike the other main cell-wall component, peptidoglycan, which forms a porous and comparatively insoluble meshwork, WTA form a highly hydrated, gel-like material that fills much of the space between peptidoglycan strands. WTA provide a soluble matrix through which all substances pass before reaching the bacterial cell membrane, and therefore affect bacterial access 